\title{
Phase Noise Characterization in Phase-Sensitive OTDR Systems
}

This paper was downloaded from TechRxiv (https://www.techrxiv.org).

\section{LICENSE}

CC BY 4.0

SUBMISSION DATE / POSTED DATE

05-10-2021/15-10-2021

\section{CITATION}

Adeel, Muhammad; Iqbal, Saeed; Muaz, Muhammad; Raza, Aadil (2021): Phase Noise Characterization in Phase-Sensitive OTDR Systems. TechRxiv. Preprint. https://doi.org/10.36227/techrxiv.16736440.v1

$\mathrm{DOI}$

10.36227/techrxiv.16736440.v1 


\title{
Phase Noise Characterization in Phase-Sensitive OTDR Systems
}

\author{
Muhammad Adeel, Saeed Iqbal, Muhammad Muaz and Aadil Raza*
}

\begin{abstract}
Coherent detected phase-OTDR (CDPO) systems are mainly used in distributed vibration sensing and these systems are preferred over the direct detected phase-OTDR (DDPO) systems due to their inherent property of mitigating a certain type of phase noise. There is a possibility that the existing nonmitigated phase noise can be controlled in CDPO systems only after properly investigating the characteristics of both the CDPOmitigated and CDPO-non-mitigated noise types. Hence, the first and crucial step, i.e, the characteristics of two different phase noise types are determined in this paper. This determination will help in providing ease about finding the real sources of both of the phase noise types and hence can help in mitigating the existing phase noise further.
\end{abstract}

Index Terms-Distributed acoustic sensing, direct detected phase-OTDR system, coherent detected phase-OTDR system, phase noise.

\section{INTRODUCTION}

$\mathbf{P}$ HASE-OTDR has gained more attention among the scientific community in the last decade. It is due to a very large scope of its use in many applications including intrusion recognition systems [1]-[3], pipeline leakage management [4], warning systems [5], [6], damage tolerance detection [7], railway line management [8], [9] and structural health monitoring [10]. These applications are preferred due to distributed nature of the phase-sensitive OTDR systems and extending the range of fiber under test (FUT) is a key research target of the distributed optical fiber sensing community. This range extension requires a significant drop in the noise level [11], [12].

The coherent detected phase-sensitive OTDR (CDPO) systems are normally preferred in most of the applications as compared to direct detected phase-OTDR (DDPO) due to less noise [13]. The phase noise is specifically engendered in phasesensitive OTDR systems due to the frequency drift and a broad linewidth of the laser source [14]. As the CDPO systems exhibit the additional phase information and therefore the phase noise in such systems are much alleviated as compared to that of DDPO systems [11].

Recently, [15], [16] have characterized different types of noise in coherent and direct detected phase-OTDR systems except characterizing the trace-to-trace fluctuation (TTF) or phase

M. Adeel is with the Centres of Excellence in Science and Applied Technologies (CESAT), Pakistan and The Hong Kong Polytechnic University, Hong Kong. e-mail: m.adeel@ connect.polyu.hk

Saeed Iqbal is with depatment of computer science at Barani Institute of Information Technology, Rawalpindi, Pakistan. email: saeed@biit.edu.pk

M. Muaz is with the National University of Science and Technology, Pakistan.email: m.muaz@cae.nust.edu.pk

*Aadil Raza is with the department of Physics at COMSATS University Islamabad, Islamabad, Pakistan. email: aadilraza@comsats.edu.pk noise within these systems. Several new sources of phase noise have been determined in [17], [18] and this whole work can only be benefited if the phase noise is properly categorized. This categorization will not only help in determining the source of each of the phase noise type but will also help in targeting the mitigation of the CDPO-non-mitigated phase noise for each type of phase-OTDR systems. In recent studies [19], [20] about phase noise mitigation has not revealed the mitigation in a specific type of phase noise. This is the first time that the phase noise in phase-sensitive OTDR is splitted into two parts and the role of CDPO systems in the removal of a complete one part of phase noise is elaborated.

A correlation-based moving processing window technique, termed matched-filtering-moving (MFM) method, is used to verify the involvement of phase noise with two different characteristics. Hence, it is verified that the CDPO systems do not mitigate the phase noise completely and this claim has become a matter of past only. The proposed claim is that the CDPO systems can completely mitigate a special type of phase noise only, termed spatially variable phase (SVP) noise or CDPO-mitigated phase noise. The characteristics of this noise behave differently at each spatial location of the received data. However, another type of noise, termed spatially constant phase (SCP) noise or CDPO-non-mitigated noise cannot be mitigated by the CDPO system due to its extremely different behavior than that of SVP noise.

The characteristics of both of these type of phase noises are evaluated in this paper. The claim that the CDPO systems partially mitigate the phase noise has been contradicted in this work by providing a proof that such systems remove the SVP part of the phase noise completely and retain the SCP part of phase noise within the data. Further clues were provided that the DDPO systems were unable to remove neither SVP nor SCP parts of the noise data which is the only reason such systems exhibit more noise than that of CDPO systems.

The exact sources that are responsible for the split of phase noise in two parts is still unknown. However, such a clear division is important with respect to the further improvement in CDPO systems in future work. This may involve finding the true reasons of generation of these two noise sources. Hence, this can help in mitigation of SCP noise which is still a major part of noise in CDPO systems. Moreover, the amount of noise due to either of the phase noises can be quantified with the help of the proposed investigation work. 


\section{EXPERIMENTAL SETUPS AND DESCRIPTION OF BASIC NOISE}

The two basic configurations of the phase-sensitive OTDR system are DDPO and CDPO. However, there are many derived complicated configurations of $\phi$-OTDR which are designed for the enhancement of fiber under test (FUT) [21][28] and spatial resolution (SR) [27]-[30]. Hence, improving the basic $\phi$-OTDR system is a crucial step which in turn adds the improvement in all the derived configurations. These two basic $\phi$-OTDR system configurations are shown in Fig. 1

The semiconductor optical amplifier (SOA) was used as the modulator and a function generator was used to provide the pulses of width $480 \mathrm{~ns}$ with a pulse repetition rate of $10 \mathrm{KHz}$. The purpose of using SOA is the provision of best possible extinction ratio to avoid the additional noise effects in phase noise of the system.

Both the configurations in Fig. 1 were common in all aspects except the use of photo-detectors. These involve the use of NKT laser source (Model: BASIK X15) with the linewidth less than $100 \mathrm{~Hz}$ and a frequency drift of $200 \mathrm{KHz} / \mathrm{sec}$. Moreover, the FUT of $7.5 \mathrm{~km}$ was used in both the configurations and the signals were acquired with the data acquisition rate of $250 \mathrm{MHz}$.

To avoid any difficulty in determining the starting point of a data-trace, the same pulses used by the function generator to modulate the light signals were employed as the external clock to the data acquisition device. The received data is the result of an interaction between Rayleigh backscattered signals within the pulse-width and can be shown in the following relation.

$$
\begin{aligned}
\gamma_{m} & =|E|^{2}=\sum_{n=1}^{Q-1}|r|^{2} \\
& +2 \sum_{v=1}^{N-1} \sum_{u=v+1}^{U}\left|r_{u}\right|\left|r_{v}\right| \cos \left(\phi_{u}-\phi_{v}-\phi_{n s}\right),
\end{aligned}
$$

where $N$ represents the particles of the optical fibre and the light signals are backscattered from these $N$ particles. The angles $\phi_{v}$ and $\phi_{u}$ represent all the angles from the region of perturbation and the angle $\phi_{n s}$ represents the phase noise. The subscript $m$ represents individual points at the spatial domain and a group of these spatial points under a single pulse traversing along the whole fiber represents a data trace. The intensity $|r|^{2}$ fluctuates randomly and determines the magnitude of the overall noise. The phase information in CDPO system plays a vital role in determining the accurate estimation of a change in phase of the received backscattered signals and avoids the SVP noise type. Clues to avoid of SVP noise are presented in this paper.

A group of data traces makes a matrix and a data vector against a single spatial point is defined as spatial data vector (SDV). After taking a difference of the traces represented in Eq. 1, the analytical expression becomes as follows

$$
\begin{aligned}
\delta_{m} & =2 \sum_{v=1}^{Q-1} \sum_{u=Q}^{U}\left|r_{v} \| r_{u}\right|\left[\cos \left(\phi_{v}-\phi_{u}-\phi_{n s}\right)\right. \\
& \left.-\cos \left(\phi_{v}-\phi_{u}-\phi_{n s}^{\prime}-\phi_{p}[m]\right)\right] .
\end{aligned}
$$

The angle $\phi_{p}$ shows the phase change due to the applied perturbation at each $m^{t h}$ spatial location whereas the angles $\phi_{n s}$ and $\phi_{n s}^{\prime}$ impersonate the applied perturbation even if there is no perturbation and can be regarded as the phase noise. By neglecting any applied perturbation, the probability density function (PDF) of these noises are depicted in Fig. 2 for each configuration of the two phase-sensitive OTDR systems. Fig. 2 shows the effect of noise is more prominent in case of DDPO systems as compared to CDPO systems. However, the details of the categorization of phase noise types in CDPO cannot be depicted from this figure.

\section{PROPOSED WORK}

The previous section provided a detailed description of phase noise within both CDPO and DDPO systems. The literature revealed the fact about the additional phase information in CDPO systems which distinguish such systems from the DDPO regarding a lower noise. The literature still lack the additional information about the type of noise that has been mitigated by the CDPO systems. It is therefore important to provide a detail description about the type of noises in phasesensitive OTDR systems. It may elaborate why the additional phase information in CDPO systems cannot eliminate the whole phase-noise in the differential SDVs. The MFM method is introduced to verify the two different characteristics of the phase noise which exist within phase-sensitive OTDR systems. These characteristics clearly differentiate between SCP and SVP noises.

\section{A. MFM method}

The basic terminologies need to be determined before jumping towards the MFM method. A data trace is one that represents a linear dimensional vector of the whole FUT after the modulated pulse is passed through it. Each element of this vector represents a spatial location. Multiple data traces need to be acquired before the creation of spatial data vectors, abbreviated $\rho_{m}$. Differentiating one data trace from the adjacent one gives rise to differential SDV defined as $\Delta \rho_{m}$. If a single $\Delta \rho$ at a spatial location $m$ is considered to be the time domain data at a specified spatial location and the length of processing window is considered to be the width of the window that processes a set of SDVs in a single drag. The direction of movement of the processing window is shown in Fig. 3. Due to very high sampling rate $(250 \mathrm{MHz})$ of data-acquisition card, the spatial domain lies in the fastaxis whereas a relative low sampling rate $(10 \mathrm{KHz})$ of PRR in the time-domain exist within the slow-axis. The processing window is moved in the right direction, as depicted in Fig. 3. For each drag, we get a single computed value and a plot in one dimensional view and such figure plots will be explained further in next sub-sections.

We know the length of $\operatorname{SDV}\left(L_{\rho}\right)$ depends upon the total number of acquired data traces such that $2 \leq L_{\rho} \leq\left(L_{S R}+L_{S T}\right)$ where $L_{S R}$ is the spatial length that corresponds to the spatial resolution of the injected pulse-width and $L_{S T}$ corresponds to the stretched length of the fibre where $L_{S T}=0$ at the spatial points of no perturbation. 


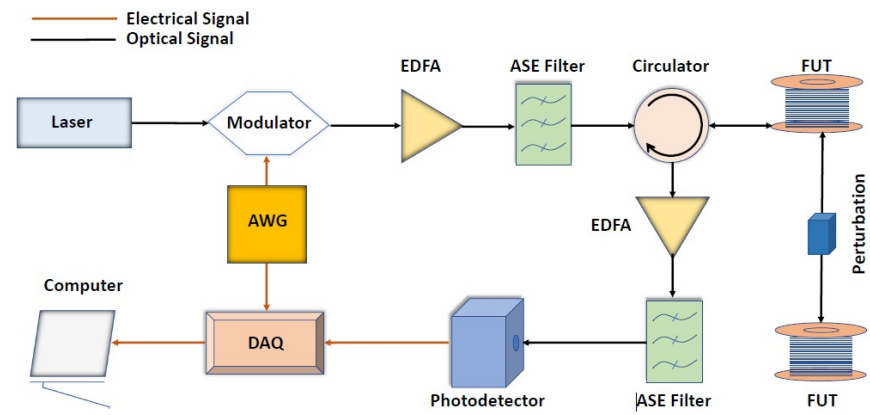

(a)

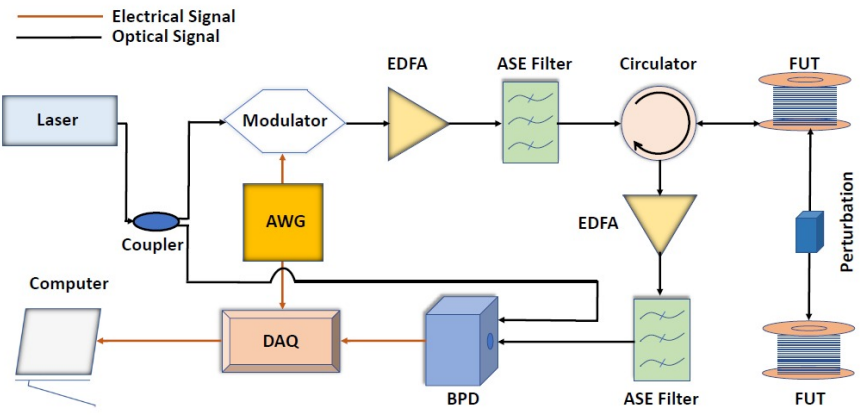

(b)

Fig. 1: $\phi$-OTDR configurations for (a) DDPO System (b) CDPO System.

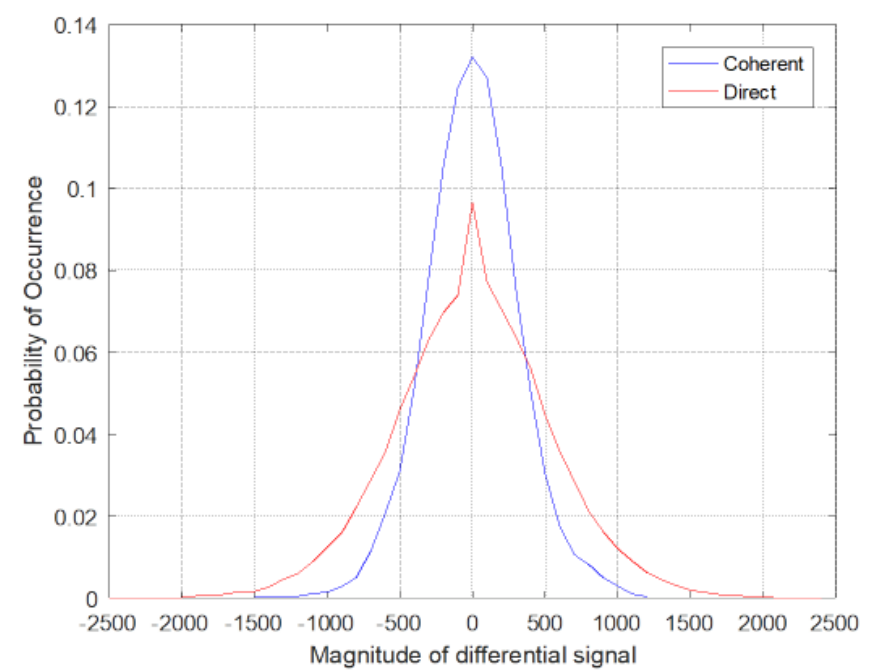

Fig. 2: Probability density of noise at non-perturbed regions for both CDPO and DDPO configuration setups.

The processing window takes in the vectors $\Delta \rho_{m}$. Each element of the vector $\Delta \rho_{m}$ is represented by $\Delta \rho(n)$ where $n$ represents the index of a single SDV and can be defined

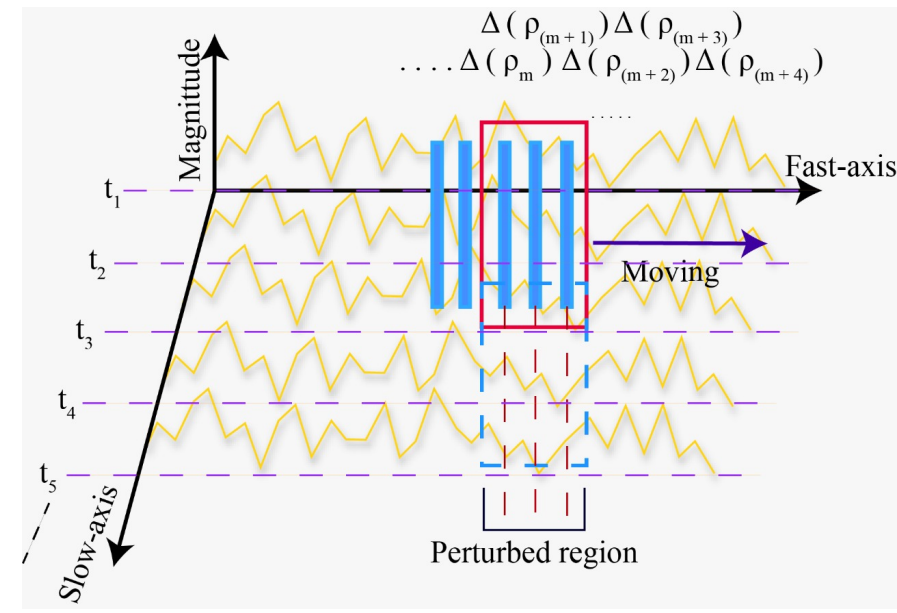

Fig. 3: Demonstration of application of MFM technique. as $n=1,2,3 \ldots N, \forall n$ and performs the correlation of each vector $\Delta \rho_{m}$ with its adjacent counterpart $\Delta \rho_{m-1}$. We know that for an ideal laser source with the zero linewidth and the modulator with unlimited extinction ratio provides no change in the vector $\Delta \rho_{m}$ which implies

$$
\delta\left\lfloor\Delta \rho_{m}\right\rfloor=\Delta \rho_{m}(n)-\Delta \rho_{m}(n-1)=0, \forall n
$$

However, in a real scenario, $\delta\left\lfloor\Delta \rho_{m}\right\rfloor \neq 0$ due to phase noise. We need to know if $\delta\left\lfloor\Delta \rho_{m}\right\rfloor$ has the same effect as $\delta\left\lfloor\Delta \rho_{m+m^{\prime}}\right\rfloor$ where $m^{\prime}$ is any random number within the range $2 \leq L_{\rho} \leq\left(L_{S R}+L S T\right)$. For this, the correlation operator is applied to determine the value $C\left\lfloor\Delta \rho_{m}, \Delta \rho_{m+m^{\prime}}\right\rfloor$ where $C[X, Y]$ represents the correlation among any two vectors $X$ and $Y$.

\section{B. MFM method as applied to DDPO systems}

A prove of the existence of both SVP and SCP noise effects have been provided with the help of DDPO systems. We consider the system in Fig. 1 (a) and investigate if the effect of $C\left\lfloor\Delta \rho_{m}, \rho_{m-1}\right\rfloor$ is comparable with that of $C\left\lfloor\Delta \rho_{m}, \Delta \rho_{m+m^{\prime}}\right\rfloor$. Fig. 4(a) clearly indicates $\delta\left\lfloor\Delta \rho_{m}\right\rfloor \neq$ $\delta\left\lfloor\Delta \rho_{m+m^{\prime}}\right\rfloor$ in the non-perturbed region after the application of MFM method where the operator $\delta[x]$ represents a change in differential SDV due to the phase noise or applied perturbation. Fig. 4 (a) shows the non-perturbed region around [0-180] and [320-400] samples whereas the perturbed region is around [181-319] samples. Fig. 4 (b) represents the result after applying MFM technique on the received traces.

Initially, a proof of the existence of SVP and SCP noise effects from the non-perturbed region is required. The non-perturbed region in Fig. 4 (a) demonstrates that $\Delta \rho_{m}$ changes differently with respect to the vector $\Delta \rho_{m-1}$ which shows the existence of SVP noise. This is due to the fact that the random changes due to noise in one SDV is not the same when compared to a change in another SDV. The average value of 0.3 in Fig. 4 (b) is a proof of $1>1 / M \sum_{m=1}^{M} \Delta \rho_{m}>0$. A value less than 1 and greater than zero represents both the SVP and SCP noise effects. With a value 1 , it represents the negligible SVP noise effect and only the presence of SCP noise whereas a value 


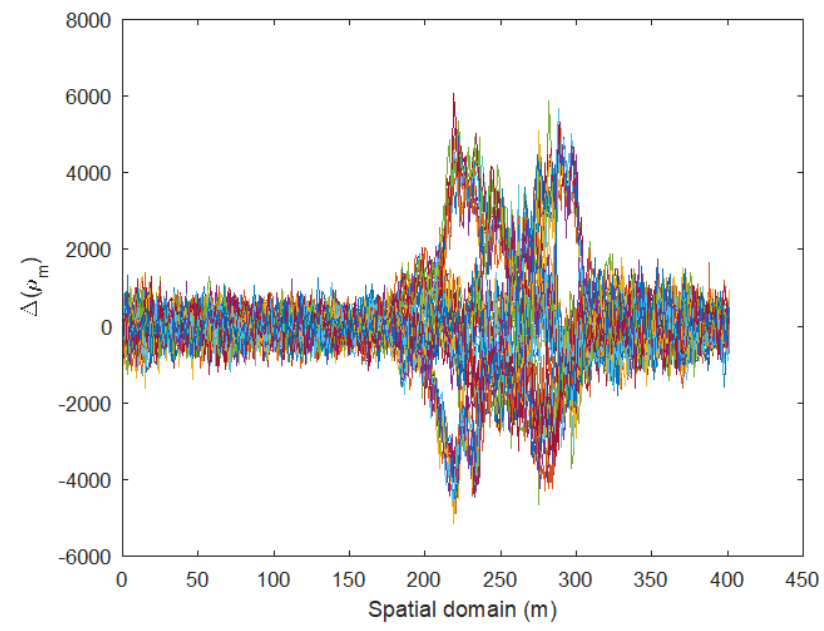

(a)

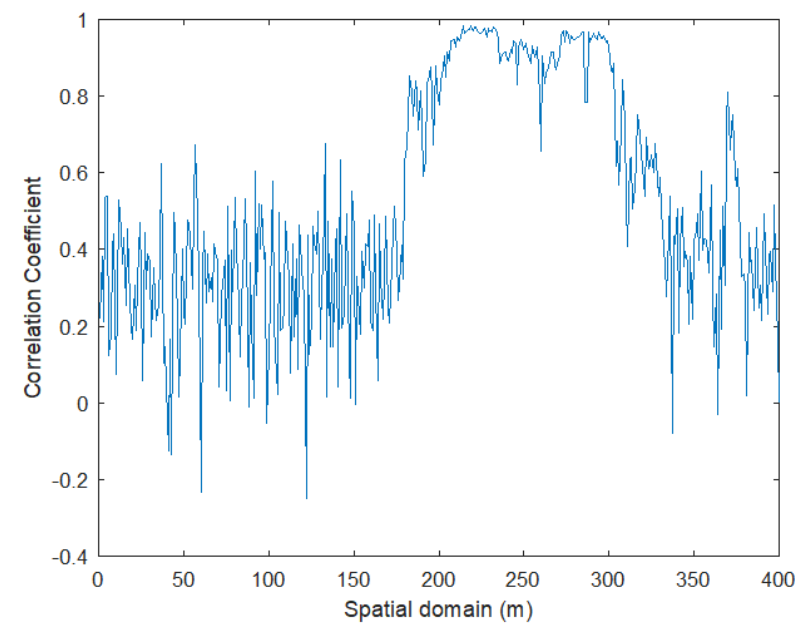

(b)

Fig. 4: Perturbed and non-perturbed regions demonstration for DDPO system using (a) Adjacent trace differentiating method (b) MFM method.

closed to 0 represents the presence of SVP noise only. For-instance, we consider two different spatial locations within the non-perturbed region. By taking a difference of differential SDV in slow-axis, we get the two relations as expressed in Eq. 4 and Eq. 5:

$$
\omega_{m}(n)=\Delta \rho_{m}(n)-\Delta \rho_{m}(n-1)
$$

and

$$
\omega_{m}^{\prime}(n)=\Delta \rho_{m^{\prime}}(n)-\Delta \rho_{m^{\prime}}(n-1) .
$$

By assuming the SVP noise to be normal distributed, this noise under the differential SDV can be defined as follows

$$
\mathcal{N}\left(\mu_{\text {svp }}, \sigma_{\text {svp }}\right)=\omega_{m}-\omega_{m^{\prime}} \neq 0 .
$$

A single disturbance due to the frequency drift of the laser source creates the same effect in all differential SDVs within the length $L_{\rho}$. This constant effect under the whole length $L_{\rho}$ can be defined as SCP noise. Assuming SCP noise to be normally distributed, the total noise of the SDV vectors for non-perturbed region in DDPO systems implies

$$
\begin{array}{r}
\rho_{m}=\int \mathcal{N}\left(\mu_{s c p}, \sigma_{s c p}\right) d t+\int \mathcal{N}\left(\mu_{\text {svp }}, \sigma_{s v p}\right) d t \\
+\mathcal{N}\left(\mu_{n p n}, \sigma_{n p n}\right)
\end{array}
$$

where $\mathcal{N}\left(\mu_{n p n}, \sigma_{n p n}\right)$ does not belong to phase noise or in other words, it belongs to other environmental and system level noise. As we know $\mathcal{N}\left(\mu_{n p n}, \sigma_{n p n}\right)$ does not belong to trace-to-trace fluctuation noise and can be eliminated after differentiating Eq. 7

$$
\frac{\partial}{\partial t}\left(\rho_{m}\right)=\mathcal{N}\left(0, \sigma_{s c p}\right)+\mathcal{N}\left(0, \sigma_{s v p}\right)
$$

For any phase noise effect or the effect of a real perturbation, we take a second differential and hence for the DDPO system we get the following relation

$$
\frac{\partial^{2}}{\partial t^{2}}\left(\rho_{m}\right)=\frac{\partial}{\partial t}\left[\mathcal{N}\left(0, \sigma_{s c p}\right)+\mathcal{N}\left(0, \sigma_{s v p}\right)\right] .
$$

The first differential in Eq. 8 represents the differential SDVs whereas the second differential in Eq. 9 represents the change in differential SDVs due to the external noise or applied perturbation.

The non-perturbed region in Fig. 4 (b) is proved to exhibit both the SVP and SCP noise effects. However, for an alternate proof of these noise effects in the Rayleigh backscattered signals of DDPO, we refer to the perturbed region. In principle, all the differential SDVs must change proportionally for any change in the Rayleigh backscattered signal to prove the existence of SCP noise. It can be seen from Fig. 4 (b) that after the application of external perturbation, all the SDVs change proportionally within the perturbed region. A perturbed region can be defined as the distance reflecting the spatial resolution such that the effect of perturbation lies within a distance measuring the spatial resolution. With the applied perturbation, the differential SDVs in the perturbed region change significantly which provides an analogous effect of SCP noise. After MFM technique is applied, the analogous effect of SCP noise becomes visible as shown in Fig. 4 (b). It can be clearly seen in Fig. 4 (b) that the applied perturbation exhibits a significant change at each differential SDV where the change due to SVP noise is negligible as compared to the amplitude of the applied perturbation. Hence, in the perturbed region, it can be seen that $\left.\delta\left\lfloor\Delta \rho_{m}\right\rfloor \sim \delta \mid \Delta \rho_{m+m^{\prime}}\right\rfloor$ for almost all differential SDVs except fewer spatial locations. This provides a clue of the existence of SCP noise within DDPO systems with a minimal effect of SVP noise. 


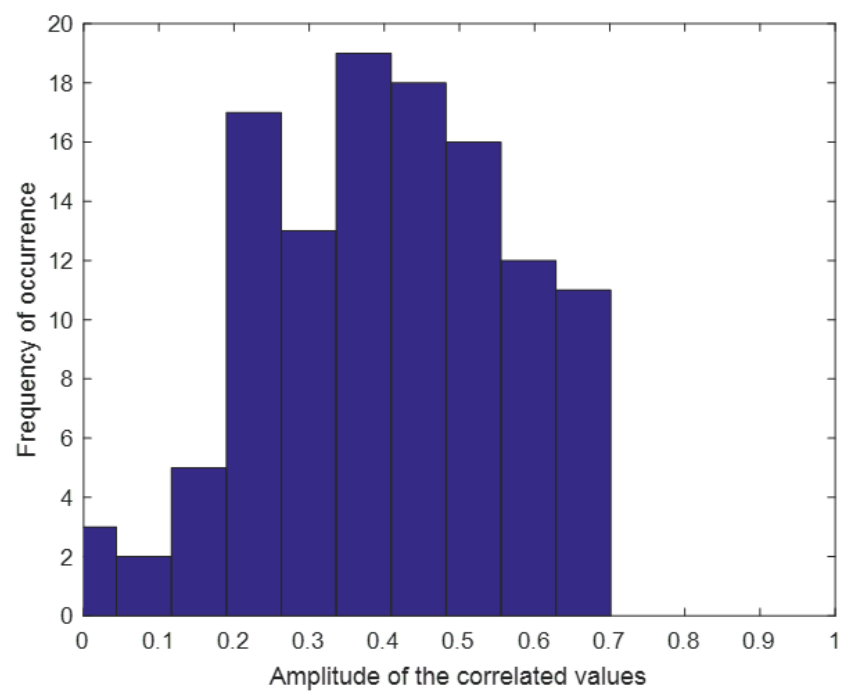

(a)

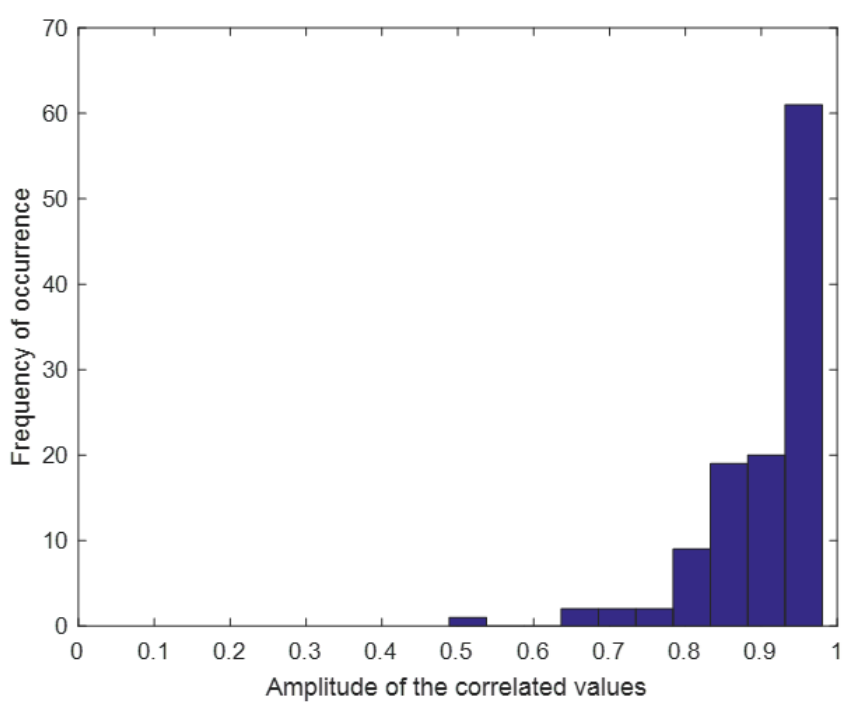

(c)

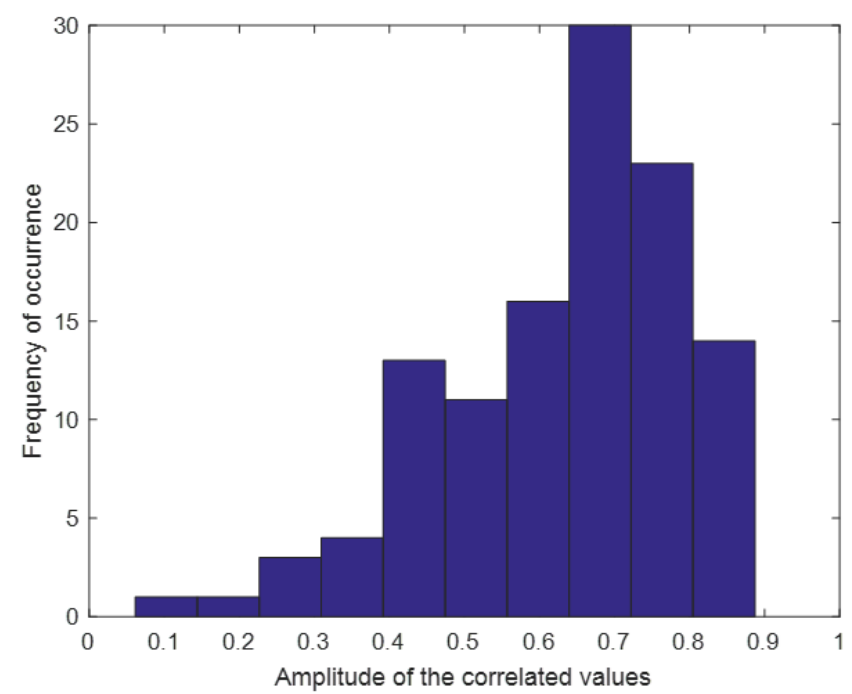

(b)

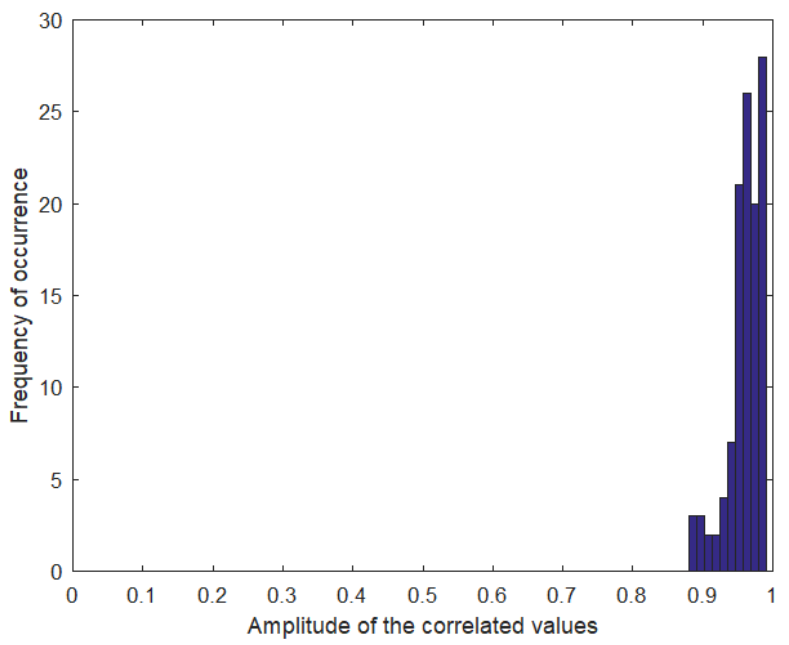

(d)

Fig. 5: Histogram of perturbed regions by applying voltage intensities to PZT with (a) $1 \mathrm{v}$ (b) $2 \mathrm{v}$ (c) $5 \mathrm{v}$ (d) $10 \mathrm{v}$

To consolidate the claim of the existence of SCP noise with the help of analogous method of exceeding SCP noise effect within the perturbation region, different intensities of the external applied perturbations are used. For this purpose, a PZT was used with different applied intensities. A peak-peak voltage of $1 \mathrm{v}, 2 \mathrm{v}, 5 \mathrm{v}$ and $10 \mathrm{v}$ are applied to the PZT and the only perturbed region data is considered. After plotting the histogram, as in Fig. 5 (a)-(d), it can be seen that after applying MFM process, the correlated values of the samples within the perturbed region shifts to the right side with the increase in applied voltages. For the lower applied voltages, low correlation is observed among the samples. This proves that if the analogous effect of SCP noise is more dominant than SVP noise, the correlation values defined in histogram of Fig. 5 shifts to the extreme right. For the analogous effect of SCP noise lower as compared to SVP, the overall correlation decreases. It means for a high value of applied intensity, most of the differential SDV vectors change proportionally due to a high dominant effect of SCP noise over the SVP. However, with the less applied intensity, the effect of SVP noise is not much lowered and hence there is a variation between all the differential SDVs in the perturbed region.

\section{MFM method as applied to CDPO systems}

We consider the configuration of phase-sensitive OTDR system in Fig. 1 (b). The MFM method is applied on CDPO system to compare the effect of $\delta\left\lfloor\Delta \rho_{m}\right\rfloor$ with that of $\delta\left[\Delta \rho_{m+m^{\prime}}\right]$. An interesting fact is observed about this processing that with CDPO system $\delta\left\lfloor\Delta \rho_{m}\right\rfloor \sim \delta\left\lfloor\Delta \rho_{m+m^{\prime}}\right]$. It means that in CDPO systems, the effect of SVP noise is extremely lowered. 


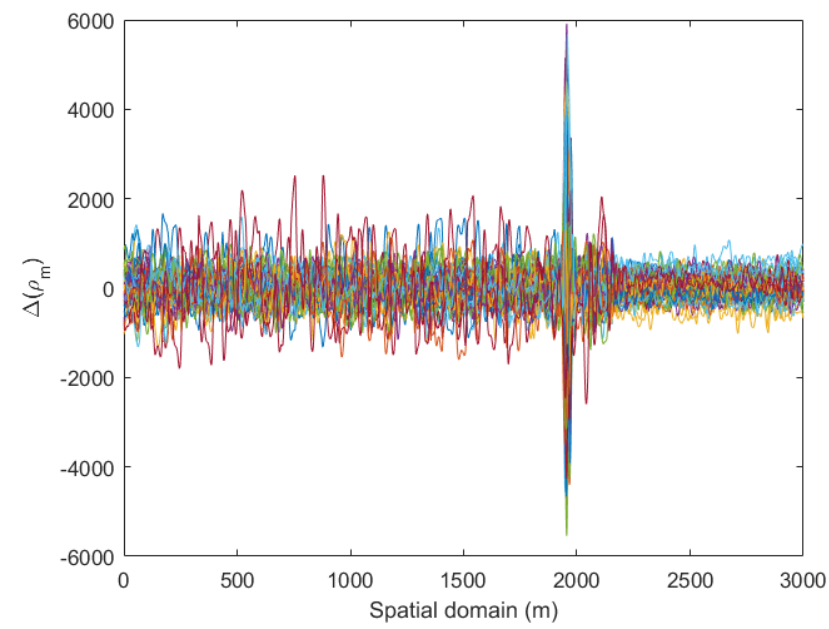

(a)

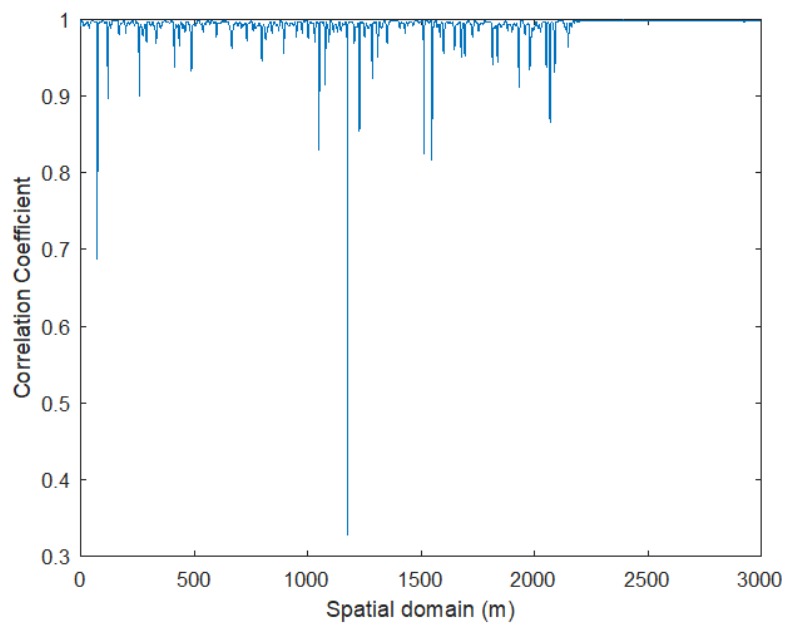

(b)

Fig. 6: Perturbed and non-perturbed regions demonstration for CDPO system using (a) Adjacent trace differentiating method (b) MFM method.

For the signal $\rho_{m}$, Eq. 4 and Eq. 5 are used to get the relation for CDPO system in its non-perturbed region and is given below,

$$
\mathcal{N}\left(\mu_{\text {svp }}, \sigma_{\text {svp }}\right)=\omega_{m}-\omega_{m^{\prime}}=0
$$

It means the signal $\rho_{m}$ does not exhibit the SVP noise effect and hence the perturbed region of the signal can be modeled as follows

$$
\begin{array}{r}
\rho_{m}=\int \mathcal{N}\left(\mu_{s c p}, \sigma_{s c p}\right) d t \\
+\mathcal{N}\left(\mu_{n p n}, \sigma_{n p n}\right) .
\end{array}
$$

For the differential SDVs, the following expression is obtained and it shows no SVP noise effect.

$$
\left.\frac{\partial}{\partial t}\left(\rho_{m}\right)=\mathcal{N}\left(0, \sigma_{s c p}\right)\right)
$$

For any perturbation or TTF noise effect, the differential SDV signals in CDPO implies,

$$
\frac{\partial^{2}}{\partial t^{2}}\left(\rho_{m}\right)=\frac{\partial}{\partial t}\left[\mathcal{N}\left(0, \sigma_{s c p}\right)\right] .
$$

After applying MFM technique on Eq. 13 and considering only two spatial locations $m$ and $m^{\prime}$, we get

$$
\mathrm{C}\left\{\frac{\partial^{2}}{\partial t^{2}}\left(\rho_{m}\right), \frac{\partial^{2}}{\partial t^{2}}\left(\rho_{m^{\prime}}\right)\right\}=K_{s c p}
$$

where $k_{S C P}$ is a constant and its theoretical value is 1 . This claim given in Eq. 14 has been demonstrated in Fig. 6.

Fig. 6 (a) demonstrates the data with a portion showing the non-perturbed region and a perturbed region. The processing window in case of CDPO system was considered with the same condition as that of DDPO as, $2 \leq L_{\rho} \leq\left(L_{S R}+L_{S T}\right)$. After applying MFM technique, the elements of adjacent differential SDVs change proportionally with respect to eachother as shown in Fig. 6 (b). A highest correlation value except at few minor points can be observed in Fig. 6 (b) which obviously shows that CDPO induce negligible noise in the received data traces. It also demonstrates that the perturbed and non-perturbed regions provide the same level at $K_{s c p}$ which provides a proof of the elimination of SVP noise only and that the SCP noise exists which give the same results in both regions.

\section{CONCLusion}

A number of noise effects are added on the back-reflected signals of the phase-sensitive OTDR system. Most of these noise effects are simply eliminated after subtracting the adjacent data traces. Moreover, the phase noise was splitted in two different types and the claim is proved with the help of results. The MFM technique is a tool to show the characteristics of SCP and SVP noise effects. The experimental data reveals that the phase noise type, termed SVP, was eliminated completely with the help of extra phase information of the CDPO system. This provides a proof that the phase information in CDPO systems is not useful for the mitigation of SCP noise. The results from MFM method also shows that there is a coexistence of SVP and SCP noise effects within the nonperturbed region of DDPO system and these types of noise are not mitigated due to the lack of phase information. The perturbed region created the effect analogous to a very high intensity SCP noise and hence the existence of both noise types are proved to co-exist within the DDPO systems. Another phase noise type, termed SCP noise, is neither mitigated by CDPO nor by DDPO systems.

This research work can provide a baseline to the researcher to evaluate the sources responsible for the creation of SCP noise effect as the detailed description about the characteristics of this noise is provided in this work. Therefore, solutions that 
specifically target the SCP noise effect will provide more better results.

\section{ACKNOWLEDGMENT}

This work is supported by COMSATS University Islamabad (CUI), Islamabad, Pakistan, under research productivity funds CUI/ORIC-PD/2021. The authors would like to thank Prof. Chao Lu of The Hong Kong Polytechnic University for providing support of lab access within the campus for acquiring the desired data. The authors are also thankful to the university administration for this support.

\section{REFERENCES}

[1] M. Adeel, C. Shang, D. Hu, H. Wu, K. Zhu, A. Raza, and C. Lu, "Impact-based feature extraction utilizing differential signals of phasesensitive OTDR," Journal of Lightwave Technology, vol. 38, no. 8, pp. 2539-2546, 2020.

[2] Z. He and Q. Liu, "Optical fiber distributed acoustic sensors: A review," Journal of Lightwave Technology, vol. 39, no. 12, pp. 3671-3686, 2021.

[3] M. Adeel, C. Shang, K. Zhu, and C. Lu, "Nuisance alarm reduction: Using a correlation based algorithm above differential signals in direct detected phase-OTDR systems," Opt. Express, vol. 27, no. 5, pp. 7685-7698, Mar 2019. [Online]. Available: http://www.osapublishing.org/oe/abstract.cfm?URI=oe-27-5-7685

[4] M.-T. Hussels, S. Chruscicki, D. Arndt, S. Scheider, J. Prager, T. Homann, and A. K. Habib, "Localization of transient events threatening pipeline integrity by fiber-optic distributed acoustic sensing," Sensors, vol. 19, no. 15, pp. 1424-8220, Jul 2019. [Online]. Available: https://www.ncbi.nlm.nih.gov/pmc/articles/PMC6696037/

[5] F. Peng, H. Wu, X.-H. Jia, Y.-J. Rao, Z.-N. Wang, and Z.P. Peng, "Ultra-long high-sensitivity $\phi$-OTDR for high spatial resolution intrusion detection of pipelines," Opt. Express, vol. 22, no. 11, pp. 13804-13810, Jun 2014. [Online]. Available: http://www.opticsexpress.org/abstract.cfm?URI=oe-22-11-13804

[6] M. Adeel, J. Tejedor, J. Macias-Guarasa, and C. Lu, "Improved perturbation detection in direct detected $\phi$-OTDR systems using matched filtering," IEEE Photonics Technology Letters, vol. 31, no. 21, pp. 16891692, 2019.

[7] Z. Qin, L. Chen, and X. Bao, "Continuous wavelet transform for non-stationary vibration detection with phase-OTDR," in OFS2012 22nd International Conference on Optical Fiber Sensors, Y. Liao, W. Jin, D. D. Sampson, R. Yamauchi, Y. Chung, K. Nakamura, and Y. Rao, Eds., vol. 8421, International Society for Optics and Photonics. SPIE, 2012, pp. 1537 - 1540. [Online]. Available: https://doi.org/10.1117/12.975025

[8] F. Peng, N. Duan, Y.-J. Rao, and J. Li, "Real-time position and speed monitoring of trains using phase-sensitive OTDR," IEEE Photonics Technology Letters, vol. 26, no. 20, pp. 2055-2057, 2014.

[9] Z. Wang, B. Lu, H. Zheng, Q. Ye, Z. Pan, H. Cai, R. Qu, Z. Fang, and H. Zhao, "Novel railway-subgrade vibration monitoring technology using phase-sensitive OTDR," in 2017 25th Optical Fiber Sensors Conference (OFS), 2017, pp. 1-4.

[10] M. Soriano-Amat, D. Fragas-Sánchez, H. F. Martins, D. VallespínFontcuberta, J. Preciado-Garbayo, S. Martin-Lopez, M. GonzalezHerraez, and M. R. Fernández-Ruiz, "Monitoring of a highly flexible aircraft model wing using time-expanded phase-sensitive OTDR," Sensors, vol. 21, no. 11, 2021. [Online]. Available: https://www.mdpi.com/1424-8220/21/11/3766

[11] A. H. Hartog, An Introduction to Distributed Optical Fibre Sensors (1st ed.). CRC Press, 2017.

[12] M. Adeel, J. Tejedor, J. Macias-Guarasa, C. Shang, and C. Lu, "Segregating the true perturbation position from ghost energy points region in $\phi$-OTDR systems," Opt. Express, vol. 28, no. 3, pp. 2699-2713, Feb 2020. [Online]. Available: http://www.opticsexpress.org/abstract.cfm?URI=oe-28-3-2699

[13] P. Gysel and R. Staubli, "Statistical properties of rayleigh backscattering in single-mode fibers," Journal of Lightwave Technology, vol. 8, no. 4, pp. $561-567,1990$.

[14] M. Adeel and C. Lu, "Signal processing techniques for direct-detected f-otdr systems," Ph.D. dissertation, The Hong Kong Polytechnic University, Hong Kong, 2020.
[15] X. Lu and K. Krebber, "Characterizing detection noise in phase-sensitive optical time domain reflectometry," Opt. Express, vol. 29 , no. 12, pp. 18791-18 806, Jun 2021. [Online]. Available: http://www.opticsexpress.org/abstract.cfm?URI=oe-29-12-18791

[16] A. E Alekseev, Y. A. Tezadov, and V. T. Potapov, "Intensity noise limit in a phase-sensitive optical time-domain reflectometer with a semiconductor laser source," Laser Physics, vol. 27, no. 5, p. 055101, May 2017.

[17] G. Yang, X. Fan, S. Wang, B. Wang, Q. Liu, and Z. He, "Long-range distributed vibration sensing based on phase extraction from phasesensitive OTDR," IEEE Photonics Journal, vol. 8, no. 3, pp. 1-12, 2016.

[18] J. Pastor-Graells, M. R. Femández-Ruiz, H. F. Martins, A. Garcia-Ruiz, S. Martin-Lopez, and M. Gonzalez-Herraez, "Impact of the laser phase noise on chirped-pulse phase-sensitive OTDR," in 2017 25th Optical Fiber Sensors Conference (OFS), 2017, pp. 1-4.

[19] P. P. P. F. Y. G. C. Z. C. N. Shao Yuying, Liu Huanhuan and W. Tingyun, "Distributed vibration sensor with laser phase-noise immunity by phaseextraction $\phi$-OTDR," Applied Physics Express, vol. 9, no. 3, pp. 223229, Sep. 2019.

[20] Z. Ma, J. Jiang, S. Wang, K. Liu, S. Wang, T. Xu, Y. Zhang, W. Chen, P. Niu, G. Liang, and T. Liu, "Phase drift noise suppression for coherentOTDR sensing based on heterogeneous dual-sideband LFM pulse," Applied Physics Express, vol. 13, no. 8, p. 082002, Aug. 2020.

[21] H. F. Martins, S. Martín-López, P. Corredera, M. L. Filograno, O. Frazão, and M. Gonzalez-Herráez, "Phase-sensitive optical time domain reflectometer assisted by first-order raman amplification for distributed vibration sensing over gt;100 km," Journal of Lightwave Technology, vol. 32, no. 8, pp. 1510-1518, 2014.

[22] H. F. Martins, S. Martin-Lopez, P. Corredera, J. D. Ania-Castañon, O. Frazão, and M. Gonzalez-Herraez, "Distributed vibration sensing over $125 \mathrm{~km}$ with enhanced snr using $\phi$-OTDR over a urfl cavity," Journal of Lightwave Technology, vol. 33, no. 12, pp. 2628-2632, 2015.

[23] Y.-J. Rao, J. Luo, Z.-L. Ran, J.-F. Yue, X.-D. Luo, and Z. Zhou, "Long-distance fiber-optic $\phi$-OTDR intrusion sensing system," in 20th International Conference on Optical Fibre Sensors, J. D. C. Jones, Ed., vol. 7503, International Society for Optics and Photonics. SPIE, 2009, pp. 250 - 253. [Online]. Available: https://doi.org/10.1117/12.835324

[24] Z. N. Wang, J. J. Zeng, J. Li, M. Q. Fan, H. Wu, F. Peng, L. Zhang, Y. Zhou, and Y. J. Rao, "Ultra-long phasesensitive OTDR with hybrid distributed amplification," Opt. Lett., vol. 39, no. 20, pp. 5866-5869, Oct 2014. [Online]. Available: http://www.osapublishing.org/ol/abstract.cfm?URI=ol-39-20-5866

[25] Z. N. Wang, M. Q. Fan, L. Zhang, H. Wu, D. V. Churkin, Y. Li, X. Y. Qian, and Y. J. Rao, "Long-range and high-precision correlation optical time-domain reflectometry utilizing an all-fiber chaotic source," Opt. Express, vol. 23, no. 12, pp. 15514-15520, Jun 2015. [Online]. Available: http://www.osapublishing.org/oe/abstract.cfm?URI=oe-23-12-15514

[26] F. Peng, H. Wu, X.-H. Jia, Y.-J. Rao, Z.-N. Wang, and Z.P. Peng, "Ultra-long high-sensitivity $\phi$-OTDR for high spatial resolution intrusion detection of pipelines," Opt. Express, vol. 22, no. 11, pp. 13804-13810, Jun 2014. [Online]. Available: http://www.osapublishing.org/oe/abstract.cfm?URI=oe-22-11-13804

[27] Y. Shi, H. Feng, and Z. Zeng, "A long distance phase-sensitive optical time domain reflectometer with simple structure and high locating accuracy," Sensors, vol. 15, no. 9, pp. 21 957-21 970, 2015. [Online]. Available: https://www.mdpi.com/1424-8220/15/9/21957

[28] T. Zhu, X. Xiao, Q. He, and D. Diao, "Enhancement of snr and spatial resolution in $\phi$-OTDR system by using two-dimensional edge detection method," Journal of Lightwave Technology, vol. 31, no. 17, pp. 28512856, 2013.

[29] M. Ren, D.-P. Zhou, L. Chen, and X. Bao, "Influence of finite extinction ratio on performance of phase-sensitive optical time-domain reflectometry," Opt. Express, vol. 24, no. 12, pp. 13325-13333, Jun 2016. [Online]. Available: http://www.osapublishing.org/oe/abstract.cfm?URI=oe-24-12-13325

[30] J. Hu, Q. Zhao, X. Zhang, L. Zhang, X. Zhao, L. Kang, and $\mathrm{P}$. Wu, "Photon-counting optical time-domain reflectometry using a superconducting nanowire single-photon detector," J. Lightwave Technol., vol. 30, no. 16, pp. 2583-2588, Aug 2012. [Online]. Available: http://www.osapublishing.org/jlt/abstract.cfm?URI=jlt-30-162583 


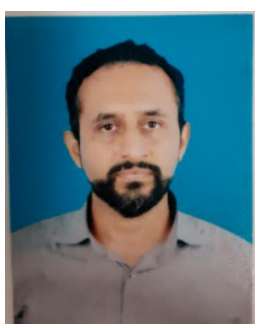

Muhammad Adeel received his Ph.D. in distributed optical fibre sensing from the Hong Kong Polytechnic Unviersity, Hong Kong. His areas of interest are distributed accoustic sensing, Electronics design, and machine learning.

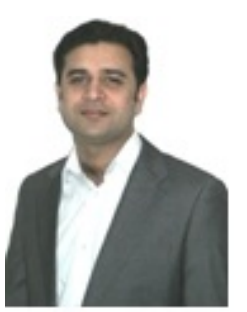

Saeed Iqbal SAEED IQBAL received his M.Sc degree in Electronics from Quaid-e-Azam University in 2005 and completed his MS in Electronic Engineering from MAJU University, Islamabad in 2010. He received his $\mathrm{PhD}$ degree in Electrical Engineering from COMSATS University, Islamabad Pakistan in 2021. Currently, he is working as an Assistant Professor at Department of Computer Science, Barani Institute of Information Technology, Rawalpindi, Pakistan. He is an active member of Next Generation Communications Research Group in the Department of Electrical and Computer Engineering, COMSATS University Islamabad. Saeed's research interests include distributed acoustic sensing, millimeter Wave technology and radio-over-fiber systems.

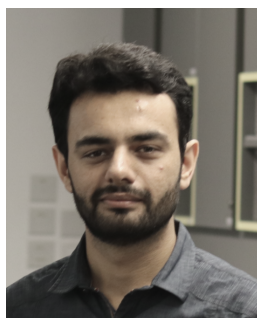

Muhammad Muaz Muhammad Muaz received his B.E. in electrical engineering from University of Engineering and Technology, Peshawar, Pakistan, and M.S. from COMSATS University Islamabad, Pakistan, in 2012 and 2014 respectively. He received his Ph.D. in statistical signal processing from the Hong Kong Polytechnic University, Hong Kong, in 2018. He has served as a Computer Engineer in 2013 at Ghulam Ishaq Khan Institute of Engineering and Sciences, Swabi, Pakistan. From 2014 to 2015, he served as a Research Associate at the University of Engineering and Technology, Peshawar, Pakistan, where he also acted as a consultant to the Pakistan Tobacco Board in using machine learning based estimation of cash crops. Currently, he is an Assistant Professor at National University of Sciences and Technology, Islamabad, Pakistan. His research interests include deep learning and signal processing.

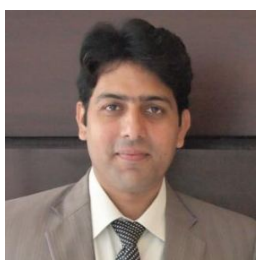

Aadil Raza received his Ph.D. in Electrical Engineering from COMSATS University Islamabad (CUI), Islamabad, Pakistan. Currently, he is serving as an assistant professor in the department of Physics, CUI, Islamabad, Pakistan. His areas of research are Electronics, Radio over Fibre systems, Free Space Optics, Chaotic Secure Communication, Data Centre Interconnect, Machine Learning and Fibre Optic Sensors. 